\title{
A Remark on Mahler's Compactness Theorem
}

\section{Citation}

Mumford, David B. 1971. A remark on Mahler's compactness theorem. Proceedings of the American Mathematical Society 28(1): 289-294.

\section{Published Version}

doi:10.2307/2037802

\section{Permanent link}

http://nrs.harvard.edu/urn-3:HUL.InstRepos:3612773

\section{Terms of Use}

This article was downloaded from Harvard University's DASH repository, and is made available under the terms and conditions applicable to Other Posted Material, as set forth at http:// nrs.harvard.edu/urn-3:HUL.InstRepos:dash.current.terms-of-use\#LAA

\section{Share Your Story}

The Harvard community has made this article openly available.

Please share how this access benefits you. Submit a story.

Accessibility 


\section{A REMARK ON MAHLER'S COMPACTNESS THEOREM}

\section{DAVID MUMFORD}

ABSTRACr. We prove that if $G$ is a semisimple Lie group without compact factors, then for all open sets $U \subset G$ containing the unipotent elements of $G$ and for all $C>0$, the set of discrete subgroups $\Gamma \subset G$ such that

(a) $\Gamma \cap U=\{e\}$,

(b) $G / \mathrm{\Gamma}$ compact and measure $(G / \Gamma) \leqq C$,

is compact. As an application, for any genus $g$ and $\epsilon>0$, the set of compact Riemann surfaces of genus $g$ all of whose closed geodesics in the Poincaré metric have length $\geqq \epsilon$, is itself compact.

Consider the following general problem: let $G$ be a locally compact topological group and let

$$
\mathfrak{M}_{G}=\{\text { the set of discrete subgroups } \Gamma \subset G\} .
$$

We would like to put a good topology on $\mathfrak{M}_{G}$ and we would like to find fairly "big" subsets of $\mathfrak{M}_{G}$ that turn out to be compact. Mahler studied the case $G=R^{n}, G / \Gamma$ compact, i.e., $\Gamma$ is lattice (cf. Cassels $[1$, Chapter 5]). In this case, the group of automorphisms of $G$, $\mathrm{GL}(n, R)$, acts transitively on the set of lattices, so that the subset $\mathfrak{M}_{G}^{C} \subset \mathfrak{M}_{G}$ of lattices can be identified as a homogeneous space under $\mathrm{GL}(n, R)$; in fact:

$$
\mathfrak{M}_{G}^{c} \cong \mathrm{GL}(n, R) / \mathrm{GL}(n, Z) .
$$

So there is only one natural topology on $\mathfrak{M}_{G}^{C}$ and Mahler's theorem states that for all $\epsilon$ and $K$ :

$$
\left\{\Gamma \subset R^{n} \mid \begin{array}{ll}
(1) & \text { if } \gamma \in \Gamma,\|\gamma\|<\epsilon \Rightarrow \gamma=0 \\
(2) & \text { volume }\left(R^{n} / \Gamma\right) \leqq K
\end{array}\right\} \text { is compact. }
$$

(Cassels [1, p. 137].)

Chabauty [2] has investigated generalizations of Mahler's theorem to general $G$ and subgroups $\Gamma$ such that measure $(G / \Gamma)<+\infty .{ }^{1} \mathrm{We}$ topologize $\mathfrak{M}_{G}$ by taking as a basis for the open sets the following:

Received by the editors April 29, 1970.

AMS 1970 subject classifications. Primary 22E40.

Key words and phrases. Discrete subgroups, Mahler's theorem.

${ }^{1}$ Although in recent years this restriction has been commonly made by people investigating automorphic functions in several variables, in the classical cases it eliminates the Fuchsian groups $\Gamma \operatorname{CSL}(2 ; R)$ of 2 nd kind, and it eliminates all Kleinian groups $\Gamma \subset S L(2 ; \boldsymbol{C})$. And $\mathfrak{M}_{G}$ seems very interesting in these cases. 


$$
\begin{array}{ll}
U \subset G \text { open, } & S_{U}=\left\{\Gamma \in \mathfrak{M}_{G} \mid \Gamma \cap U \neq \varnothing\right\}, \\
K \subset G \text { compact, } & T_{K}=\left\{\Gamma \in \mathfrak{M}_{G} \mid \Gamma \cap K=\varnothing\right\} .
\end{array}
$$

Then assuming that $G$ is not too pathological, ${ }^{2}$ Chabauty proves:

TheOREM. Let $U$ be an open neighborhood of $e, C$ a positive number. Then: $\left\{\Gamma \in \mathfrak{M}_{G} \mid \Gamma \cap U=\{e\}\right.$ and measure $\left.(G / \Gamma) \leqq C\right\}$ is compact.

This is very pretty. Its main drawback, however, is that the topology on $\mathfrak{M}_{G}$ is so weak that it is hard to deduce things from convergence in this topology. For instance if subgroups $\Gamma_{i}$ converge to $\Gamma$, one would like to know that suitable sets of generators of the $\Gamma_{i}$ converge to generators of $\Gamma$. Chabauty gives some arguments about this at the end of his paper, but I believe his reasoning there is wrong. However the results of Weil [4] and Macbeath [5] show that the topology is "strong enough" on the subset

$$
\mathfrak{M}_{G}^{C}=\left\{\Gamma \in \mathfrak{M}_{G} \mid G / \Gamma \text { compact }\right\} .
$$

Theorem (Macbeath [5, Theorems 4 and 5]). Assume that $G$ is a Lie group. ${ }^{3}$ Let subgroups $\Gamma_{i} \in \mathfrak{M}_{G}^{C}$ converge to $\Gamma \in \mathfrak{M}_{G}^{C}$. Then for $i$ sufficiently large, there exist isomorphisms of the abstract groups

$$
\phi_{i}: \Gamma \stackrel{\sim}{\rightarrow} \Gamma_{i}
$$

such that for all $\gamma \in \Gamma, \phi_{i}(\gamma) \in G$ converge to $\gamma$. Moreover there is a compact set $K \subset G$ and an open neighborhood $U \subset G$ of e such that $K \cdot \Gamma=G$, $K \cdot \Gamma_{i}=G, U \cap \Gamma=\{e\}$ and $U \cap \Gamma_{i}=\{e\}$ if $i$ is sufficiently large.

For the application that we want, Chabauty's theorem is not the right generalization of Mahler's theorem. Instead, what we want is this:

THEOREM 1. Let $G C \mathrm{GL}(n, R)$ be a semisimple Lie group without compact factors. Let $U \subset G$ be an open set containing all unipotent elements of $G$ and let $C$ be a positive number. Then

$$
\left\{\Gamma \in \mathfrak{M}_{G}^{C} \mid \Gamma \cap U=\{e\}, \text { measure }(G / \Gamma) \leqq C\right\}
$$

is compact.

Proof. This is an immediate consequence of Chabauty's theorem and Selberg's conjecture, proved recently by Kajdan and Margulis

\footnotetext{
${ }^{2} G$ satisfies the 2 nd axiom of countability, and moreover $e \in G$ has a fundamental system of neighborhoods $U_{i}$ such that measure $\left(\bar{U}_{i}-U_{i}\right)=0$. In this case, $\mathfrak{M}_{G}$ satisfies the 2 nd axiom of countability too.

${ }^{3}$ A Lie group is always assumed to be connected.
} 
[3], to the effect that a discrete subgroup $\Gamma \subset G, G$ as above, such that measure $(G / \Gamma)<+\infty$ but $G / \Gamma$ not compact, must contain nontrivial unipotent elements of $G$. Q.E.D.

Instead of invoking the difficult result of Každan and Margulis, we can prove a weaker but more explicit theorem by elementary means: Let $G \subset \operatorname{GL}(n, R)$ again be a semisimple Lie group without compact factors. Let $K \subset G$ be a maximal compact subgroup and let $X=K \backslash G$ be the associated symmetric space. Let the Killing form on $G$ induce a metric $\rho$ on $X$ as usual. Define a function $d$ on $G$ by:

$$
d(x)=\inf _{z \in X} \rho\left(z, z^{x}\right) .
$$

It is easy to see that $d$ is continuous and $d(x)=0$ if and only if when you decompose $x=x_{s} \cdot x_{u}$, ( $x_{s}$ semisimple, $x_{u}$ unipotent and $x_{s} x_{u}$ $\left.=x_{u} x_{s}\right)$, then $x_{8}$ is in a compact subgroup of $G$ or equivalently $x_{s} \in \bigcup_{y \in G} y K y^{-1}$. For all $\epsilon>0$, define an open subset of $G$ by:

$$
U_{\epsilon}=\{x \in G \mid d(x)<\epsilon\} .
$$

For all $C>0$, define a compact subset of $G$ by:

$$
K_{C}=\{x \in G \mid \rho(K \cdot x, K \cdot e) \leqq C\} .
$$

TheOREM 2. Let $n=\operatorname{dim} K \backslash G$. Then there is a constant $\gamma$ depending only on $n$ such that for all $\Gamma \in \mathfrak{M}_{G}^{C}, \epsilon>0$,

$$
\Gamma \cap U_{\mathrm{e}}=\{e\} \Rightarrow K_{C} \cdot \Gamma=G
$$

where $C=\gamma \cdot$ measure $(G / \Gamma) / \epsilon^{n-1}$. Hence for all positive $D$

$$
\left\{\Gamma \in \mathfrak{M}_{G \mid} \mid \Gamma^{C} \cap U_{\epsilon}=\{e\}, \text { measure }(G / \Gamma) \leqq D\right\}
$$

is compact.

Proof. We begin by proving:

LEMMA. Let $X$ be a compact Riemannian manifold with all sectional curvatures $R(S) \leqq 0$. There is a constant $\gamma$ depending only on $n=\operatorname{dim} X$ such that:

$\operatorname{diam}(X) \cdot(\text { length of smallest closed geodesic on } X)^{n-1} \leqq \gamma \cdot$ volume $(X)$.

Proof. Let $d=\operatorname{diam}(X)$ and let $x, y \in X$ be a distance $d$ apart. Let $\sigma$ be a geodesic from $x$ to $y$ of length $d$. Let $\eta$ be the length of the shortest closed geodesic on $X$ and construct a tube $T$ around $\sigma$ of radius $\eta / 4$ as the union of all geodesics perpendicular to $\sigma$ of length $\eta / 4$. There are 2 possibilities: either no 2 geodesics $\delta_{1}, \delta_{2}$ perpendicular to $\sigma$ of length $\eta / 4$ meet, or else some pair $\delta_{1}, \delta_{2}$ do meet. In the first 
case, we may say that the exponential map from the normal bundle $N$ to $\sigma$ in $M$ maps an $\eta / 4$-tube $T_{0}$ around the 0 -section in $N$ injectively to $M$. Then since all the sectional curvatures are $\leqq 0$, it follows that:

(*) volume $X \geqq$ volume $T \geqq$ volume $T_{0}=c_{n} \cdot(\eta / 4)^{n-1} \cdot d$

where $c_{n}$ is the volume of the unit ball in $R^{n-1}$. On the other hand, suppose 2 geodesics $\delta_{1}$ and $\delta_{2}$ meet:

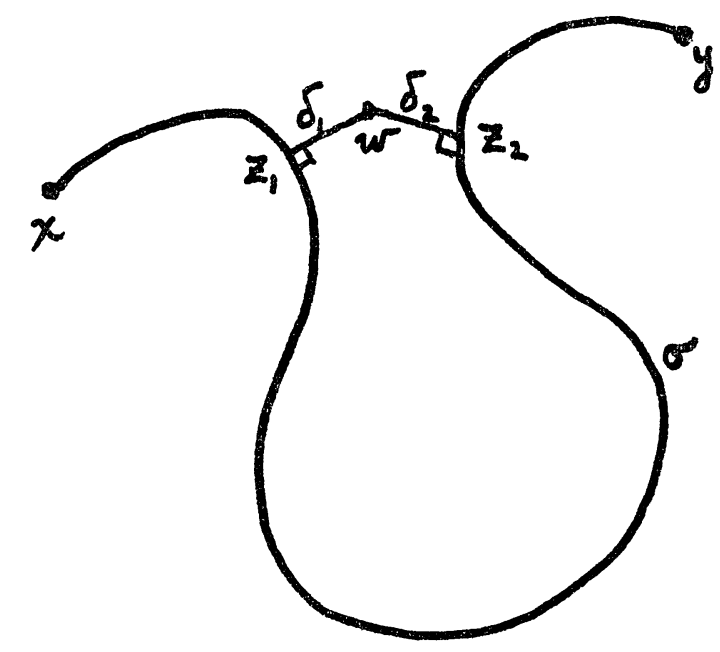

Let $z_{1}, z_{2}$ and $w$ be the points indicated in the figure and let $e$ be the distance from $z_{1}$ to $z_{2}$ along $\sigma$. Then we can go from $x$ to $y$ by going from $x$ to $z_{1}$ on $\sigma$, following $\delta_{1}$, then $\delta_{2}$ and going from $z_{2}$ to $y$ on $\sigma$. This has length $\leqq d-e+\eta / 2$, and since $\sigma$ is the shortest path from $x$ to $y, d \leqq d-e+\eta / 2$, i.e., $e \leqq \eta / 2$. But then $\delta_{1}, \delta_{2}$ and the part of $\sigma$ between $z_{1}$ and $z_{2}$ is a closed path $\tau$ of length at most $\eta$. $\tau$ is certainly not homotopic to 0 since on the universal covering space $\tilde{X}$ of $X$, the exponential from $N_{0}$ to $\tilde{X}$ is injective. Moreover, $\tau$ has corners and so is not itself a geodesic. Therefore there is a closed geodesic freely homotopic to $\tau$ of length $\langle\eta$. This contradicts the definition of $\eta$ and so the 1 st possibility must be correct. This proves $(*)$ and the lemma. Q.E.D.

We apply the lemma to the manifold $X / \Gamma$, with the metric induced from the metric $d$ on $X$. (Note that by hypothesis $\Gamma \cap U_{\epsilon}=\{e\}, \Gamma$ acts freely on $X$, so $X / \Gamma$ is a manifold.) The closed geodesics of $X / \Gamma$ are all images of geodesics in $X$ joining 2 points $x, x^{z}$, where $x \in X$, 
$z \in \Gamma$. Since $\Gamma \cap U_{\epsilon}=\{e\}$, these all have length at least $\epsilon$. It follows from the lemma that:

$$
\operatorname{diam}(X) \leqq \frac{\gamma \text { volume }(X / \Gamma)}{\epsilon^{n-1}}=\frac{\gamma \text { measure }(G / \Gamma)}{\epsilon^{n-1}}=C .
$$

Therefore the projection of $X$ onto $X / \Gamma$ maps the unit ball of radius $C$ onto $X / \Gamma$, hence $K_{C} \cdot \Gamma=G$.

Finally to prove from this that $\left\{\Gamma \in \mathfrak{M}_{G}^{C} \mid \Gamma \cap U_{\epsilon}=\{e\}\right.$ and measure $(G / \Gamma) \leqq D\}$ is compact, it suffices by Chabauty's theorem to check that if $\Gamma_{i}$ are in this set and $\Gamma_{i} \rightarrow \Gamma \in \mathfrak{M}_{G}$, then $G / \Gamma$ is also compact. But since $K_{C} \cdot \Gamma_{i}=G$ for all $i$, it follows easily that $K_{C} \cdot \Gamma=G$ too, hence $G / \Gamma$ is a quotient of $K_{C}$ and is compact. Q.E.D.

I want to apply Theorem 2 to the case $G=\operatorname{SL}(2, R) /( \pm I)$ so that $\Gamma$ is a Fuchsian group. Then $X$ is the Lobachevskian plane, and a simple calculation shows that

\section{$U_{\epsilon}=$ image of $A$ 's such that $|\operatorname{tr} A|<2 \cosh (\epsilon / 2)$}

$=$ set of elliptic and parabolic elements and those hyperbolic elements with eigenvalues $t, t^{-1}$ for which $1<t<e^{\epsilon / 2}$.

The Fuchsian groups of 1st kind which are disjoint from some $U_{\epsilon}$ are exactly those which act freely on $X$ and for which $X / \Gamma$ is compact. In this case $X / \Gamma$ is a compact Riemann surface with its Poincaré metric, $X$ is its universal covering space and $\Gamma \cong \pi_{1}(X / \Gamma)$. Moreover the map which takes an element $z \in \Gamma$ to the image mod $\Gamma$ of the shortest line segment geodesic from $x$ to $x^{z}$ in $X$ defines an isomorphism between the set of conjugacy classes in $\Gamma$ and the set of closed geodesics in $X / \Gamma$. If the conjugacy class of $\gamma$ corresponds to a geo$\operatorname{desic} \sigma$, then

$$
\cosh \frac{\text { length } \sigma}{2}=\left|\frac{\operatorname{Tr} \gamma}{2}\right| .
$$

Moreover, by the Gauss-Bonnet theorem

$$
\text { measure }(G / \Gamma)=\operatorname{area}(X / \Gamma)=\operatorname{cnst}(g-1)
$$

where $g=$ genus of $X / \Gamma$. So in this case, the lemma in Theorem 2 says:

Corollary 1. For all compact Riemann surfaces $X$ of genus $g$, $\operatorname{diam}(X) \cdot($ length of smallest geodesic on $X)$ is bounded above.

Corollary 2. For all $\epsilon>0, g \geqq 2$, the set of discrete subgroups $\Gamma \subset \mathrm{SL}(2 ; \boldsymbol{R})$ such that: 
(i) for all $\gamma \in \Gamma, \gamma \neq I,|\operatorname{Tr} \gamma| \geqq 2+\epsilon$,

(ii) $X / \Gamma$ is a compact Riemann surface of genus $g$, is compact.

CoROllary 3. Let $\mathrm{g} \geqq 2$ and let $\mathfrak{M}_{0}$ be the moduli space of compact Riemann surfaces of genus $g$ (without "marking"). For all $\epsilon>0$, the subset:

$\left\{X \in \mathfrak{M}_{g} \mid\right.$ in the Poincaré metric, all geodesics on $X$ have length $\left.\geqq \epsilon\right\}$

is compact.

(Proof. Apply Theorem 1 and Corollary 1.)

This result was my motivation for looking at these questions. I originally found a completely elementary proof of this, using the method of Theorem 2, and then finding

(a) upper bounds for the number of vertices and

(b) lower bounds for the interior and exterior angles of the Dirichlet fundamental domain for $\Gamma$ acting on $X$; but one reference leads to another and it turned out that $\{$ elem. th. $\} \subset$ Chabauty+Weil +Každan-Margulis + Macbeath.

\section{REFERENCES}

1. J. W. S. Cassels, An introduction to the geometry of numbers, Springer-Verlag, Berlin, 1959. MR 28 \#1175.

2. C. Chabauty, Limite d'ensembles et gémétrie des nombres, Bull. Soc. Math. France 78 (1950), 143-151. MR 12, 479.

3. D. A. Každan and G. A. Margulis, $A$ proof of Selberg's conjecture, Mat. Sb. 75 (117) (1968), 163-168 = Math. USSR Sb. 4 (1968), 147-152. MR 36 \#6535.

4. A. Weil, On discrete subgroups of Lie groups, Ann. of Math. (2) 72 (1960), 369384. MR $25 \# 1241$.

5. A. M. Macbeath, Groups of homeomorphisms of a simply connected space, Ann. of Math. (2) 79 (1964), 473-488. MR 28 \#4058.

Harvard University, Cambridge, Massachusetts 02138 